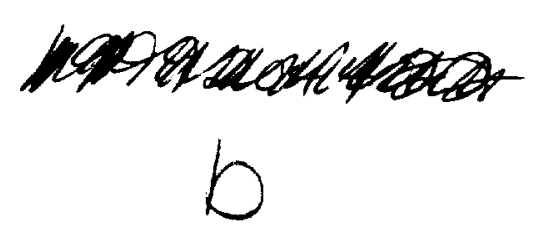

\title{
QUANTITATIVE INTERFEROMETRY IN THE SEVERE ACOUSTIC ENVIRONMENT OF RESONANT SUPERSONIC JETS
}

\author{
Carolyn R. Mercer \\ NASA Lewis Research Center \\ Cleveland, $\mathrm{OH} 44135$, USA \\ E-mail: carolyn.r.mercer@lerc.nasa.gov \\ Ganesh Raman \\ NYMA Inc., NASA LeRC Group \\ Brook Park, OH 44142 \\ E-mail: ganesh.raman@lerc.nasa.gov
}

\section{ABSTRACT}

Understanding fundamental fluidic dynamic and acoustic processes in high-speed jets requires quantitative velocity, density and temperature measurements. In this paper we demonstrate a new, robust Liquid Crystal Point Diffraction Interferometer (LCPDI) that includes phase stepping and can provide accurate data even in the presence of intense acoustic fields. This novel common path interferometer (LCPDI) was developed to overcome difficulties with the Mach Zehnder interferometer in vibratory environments and is applied here to the case of a supersonic shockcontaining jet. The environmentally insensitive LCPDI that is easy to align and capable of measuring optical wavefronts with high accuracy is briefly described, then integrated line of sight density data from the LCPDI for two underexpanded jets are presented.

\section{INTRODUCTION}

There is renewed interest in the study of supersonic jets due to advances in high speed jet propulsion, supersonic combustion, and jet noise suppression for the next generation supersonic 
commercial transport. Understanding fundamental fluid dynamic and acoustic processes for these applications requires quantitative velocity, density and temperature measurements. Thermocouples and pressure transducers can measure these parameters at discrete points, but these devices are invasive and alter the flow that they measure. Optical methods such as schlieren flow visualization have been used to get real-time, qualitative data, but it is difficult to extract quantitative data from schlieren images. Laser velocimetry, particle image velocimetry and doppler global velocimetry are used for velocity measurements but require seeding. Rayleigh scattering can be used for planar density, temperature, velocity and species measurements. Interferometry also yields density data, but to date it has been difficult to make quantitative interferometric measurements because of the high vibration environments associated with supersonic jets. This severe acoustic environment includes the three components of supersonic jet noise (Fig. 1). One of them is a discrete tone referred to as jet screech $\left(\right.$ Powell $\left.^{1}\right)$. The other two are shock-associated noise and jet mixing noise, both broadband sources. The screech resonance involves growing hydrodynamic disturbances in the shear layer that interact with the shocks to produce acoustic waves. These waves then propagate upstream and excite hydrodynamic disturbances at the nozzle exit, thus closing the resonant loop. The screech phenomenon can produce sound pressure levels in excess of $160 \mathrm{~dB}$ in the vicinity of the jet $\left(\operatorname{Raman}^{2}\right)$. This phenomenon has been linked to structural damage in aircraft engine exhaust nozzles, premature wear in protective coatings, and increased engine noise. Details of the screech process and recent advances are described in $\operatorname{Raman}^{3}$. In this paper our focus is restricted to the demonstration of a robust interferometer that can provide accurate data even in the presence of intense acoustic fields. 
An interferometer by definition requires two coherent beams to interfere, and environmental vibration and thermal disturbances affect the reference arm as well as the object arm making it difficult to isolate the information of interest.

To extract quantitative results from interferometers phase-shifting interferometry (Greivenkamp and Bruning ${ }^{5}$ ) was developed. It works on the principle that an interferogram, i.e. a sinusoidal function generated by the coherent interference of two optical waves, represents an equation with three unknowns: a DC bias, contrast, and phase. Therefore any of these three unknowns can be solved provided at least three equations exist. Multiple equations are obtained by recording interferograms with known phase differences added to the phase between the two optical waves. These equations are represented by:

$$
I_{n}(x, y)=I_{\text {avg }}(x, y)+\eta(x, y) \cos \left[\phi(x, y)+\theta_{n}\right]
$$

where $I_{n}$ is the intensity in the $n^{\text {th }}$ recorded interferogram, $I_{\text {avg }}$ is the average intensity, $\eta$ is the pattern contrast, $\phi$ is the phase difference between the two optical waves, $\theta_{n}$ is an added phase, and $(x, y)$ indicates that the variable is defined at each pixel. The phase $\phi$ is directly related to the gas density, and is therefore the quantity of interest. This phase can be calculated simply and robustly by setting $\mathbf{n}$ $=1,2,3,4,5$ and $\theta_{n}=n \pi / 2\left(\right.$ Hariharan $\left.^{6}\right) ;$ knowledge of $I_{\text {avg }}$ and $\eta$ is then not required provided they remain constant over the sequence of interferograms.

The challenge is to generate the required sequence of $\pi / 2$ phase steps. This is straightforward for conventional two beam interferometers like the Mach-Zehnder; typically a mirror in one beam is mounted on a piezoelectric device to precisely change the optical pathlength. However, two-beam 
interferometers require a reference optical path, which must not experience flow disturbances. In large facilities this reference path can be quite long, and in noisy environments it is difficult to isolate even short paths from air turbulence. Single interferograms of jets have been recorded using short exposures and small facilities (Nakamura and Iwamoto $^{7}$ ) but fringe-tracking or Fourier transform techniques must be used for data processing. Common-path interferometers ( Linnik $^{4}$ ) have been used to reduce the effect of environmental noise; they work because the reference beam travels the same path as the object beam so noise affects both beams in the same way. Since interferometry works by detecting phase differences between two beams, common disturbances do not affect the result. A modified point diffraction interferometer, the Liquid Crystal Point Diffraction Interferometer (LCPDI) was developed to both generate phase steps for automated data processing and provide the simplicity and robust performance of a common-path interferometer (see Fig. 2). This interferometer was developed for space flight experiments (Mercer and Creath ${ }^{8}$ ) and is applied here to the case of a supersonic shock-containing jet. The LCPDI and the experiments are described, a density distribution measured simultaneously using the LCPDI and a Mach-Zehnder is presented, and flow measurements from LCPDI data is presented.

\section{EXPERIMENT}

The integrated line-of-sight density of a free jet was measured simultaneously with the LCPDI and a Mach-Zehnder interferometer. The experimental apparatus is shown in Figure 3. The optical path unique to the Mach-Zehnder is shown as double-hatched lines, the optical path unique to the LCPDI is shown single-hatched, and the path common to both interferometers is shown crosshatched. A frequency-doubled diode-pumped YAG laser operating at $532 \mathrm{~nm}$ was attenuated to $5 \mathrm{~mW}$ by a neutral density filter (NDI) and directed by mirror M1 to a beam expander (EXPND) which generated 
a collimated beam. Beamsplitter BS1 transmitted $50 \%$ of the beam to form the Mach-Zehnder's reference arm. The reflected beam was directed by mirror M3 to pass just downstream of a $4.5 \mathrm{~mm}$ ID circular nozzle. Beamsplitter BS2 transmitted $50 \%$ of this beam to form the Mach-Zehnder's object beam, which was recombined, with the reference beam by beamsplitter BS3. These beams were recorded by camera CAM2. The beam reflected by BS2 was focused by a f/2.6 Cooke Triplet lens nearly onto the LCPDI. The LCPDI consists of two glass plates with a 9-micron gap filled with nematic liquid crystals and 9-micron microspheres. The LCPDI is positioned so that the focused beam illuminates one microsphere. (In practice several microspheres may be present in the focused beam.) Some of the light passes around the microsphere through the liquid crystals, and some of the light is diffracted by the microsphere forming a spherical wave. Both the diffracted reference wave and the "pass-through" object wave travel co-linearly to camera CAM1 where they combine coherently to form an interferogram.

Phase stepping is accomplished in the Mach-Zehnder by applying a sequence of DC voltages to a piezoelectric element in mirror M2. This changes the path length of the reference beam (hence its phase) while leaving the object beam unaffected. Phase stepping is accomplished in the LCPDI by applying a sequence of $\mathrm{AC}$ voltages across indium tin oxide electrodes deposited on the inner surfaces of the glass plates. This changes the refractive index of the birefringent liquid crystals and steps the phase of the light passing through them, while leaving the phase of the diffracted beam unchanged.

In both cases, the phase $\phi$ is measured by recording a sequence of 5 interferograms with an additional $\pi / 2$ radian phase step added each time. Each interferogram was recorded with an exposure 
time of $1 / 30 \mathrm{sec}$ and a delay of 2 seconds between frames to ensure that the liquid crystals were properly oriented. The data acquisition time was therefore $8.3 \mathrm{sec}$. A sequence of 5 frames was recorded with the jet on and then again with the jet off to provide a background measurement. A phase map was calculated from each of the 2 sets of 5 frames, and the two-phase distributions were then subtracted to produce a single array containing the optical phase difference between the flow and no-flow conditions. This array had the same number of elements as the recording camera; $752 \times 480$ pixels in our case. Our field of view was $20 \times 12.7 \mathrm{~mm}$ for a spatial resolution of $0.026 \mathrm{~mm} / \mathrm{pixel}$.

The gas density, $\rho$, is determined from this measured phase difference, $\Delta \phi$ by:

$$
\int_{0}^{\mathrm{L}} \rho(\mathrm{x}, \mathrm{y}) \mathrm{ds}=\frac{\lambda}{2 \pi \mathrm{K}} \Delta \phi(\mathrm{x}, \mathrm{y})+\mathrm{L} \rho_{0}
$$

where $\lambda$ is the laser wavelength, $K$ is the Gladstone-Dale constant, $L$ is the physical path length through the jet, and $\rho_{0}$ is the no-flow density. For the data presented here, $\lambda=532.0 \mathrm{~nm}, \mathrm{~K}=0.2270$ $\mathrm{cm}^{3} / \mathrm{g}\left(\right.$ Vest $\left.{ }^{9}\right), \mathrm{L}=50 \mathrm{~cm}$ and $\rho_{0}=1.205 \mathrm{~kg} / \mathrm{m}^{3}$.

If the flow under study is two-dimensional, then the above integral simplifies to $L \rho$ and the density is simply calculated. If the flow is axisymmetric, then an Abel transform can be used to calculate the radial distribution of density at each axial location. The calculation of this transform using data from the LCPDI is greatly simplified because the high spatial density of the data eliminates the need for interpolation (Kassemi et. al. ${ }^{10}$ ). If a fully three-dimensional flow is of interest, then data must be taken from multiple views and reconstructed tomographically. 


\section{EXPERIMENTAL UNCERTAINTY}

The uncertainty of the measured density is determined as follows. Since the density itself is extracted in one of the three ways outlined above, only the uncertainty in the line-of-sight integrated density is calculated. Define the relative, integrated density $Q$ as:

$$
\mathrm{Q} \equiv \int_{0}^{\mathrm{L}} \rho \mathrm{ds}-\mathrm{L} \rho_{0}
$$

The $(x, y)$ notation has been dropped for convenience. It is readily seen from equation 2 that $Q$ is proportional to $\lambda$ and $\Delta \phi$, and inversely proportional to $K$. The relative uncertainty in $Q$ is therefore equal to:

$$
\frac{\sigma_{\mathrm{Q}}}{\mathrm{Q}}=\sqrt{\left(\frac{\sigma_{\lambda}}{\lambda}\right)^{2}+\left(\frac{\sigma_{\mathrm{K}}}{\mathrm{K}}\right)^{2}+\left(\frac{\sigma_{\Delta \phi}}{\Delta \phi}\right)^{2}}
$$

For $\sigma_{\lambda}=0.1 \mathrm{~nm}, \sigma_{\mathrm{K}}=0.0001 \mathrm{~cm}^{3} / \mathrm{g}$ and the values for $\lambda$ and $\mathrm{K}$ given above, the first two squared terms equal $0.02 \%$ and $0.04 \%$, respectively. Because these uncertainties in the wavelength and GladstoneDale constant are so small, the density uncertainty approximately equals the phase measurement uncertainty for phase measurement uncertainties greater than 1 degree. Therefore, if the phase is measured to 1 degree, $\sigma_{\Delta \downarrow} / \Delta \phi=1 / 360$ and $\sigma_{Q} / Q=0.3 \%$. The relative density uncertainty is $\leq 1 \%$ for measured phase uncertainties $\leq 3.6$ degrees, and the density is known to $\leq 10 \%$ if the measured phase is determined within 36 degrees. The typical standard deviation for the measured phase is 4 degrees 
and the data presented here spanned about 6 interference cycles. Uncorrelated speckle from frame-toframe and other optical interference can cause noisy interferograms which increase the measured phase uncertainty. Note that this analysis holds for any single-pass interferometer.

\section{RESULTS}

Figure 4 shows a spark-schlieren photograph of the intense acoustic environment produced by an underexpanded jet at a fully expanded Mach number $\left(\mathrm{M}_{\mathrm{j}}\right)$ of 1.4. A larger scale rectangular nozzle with exit dimensions of $8.89 \mathrm{~cm} \times 0.635 \mathrm{~cm}$ was used for this visualization. The view shown in Figure 4 is that of the narrow dimension of the nozzle. The oscillatory motion of the entire jet and feedback shocks in the nearfield is clearly visible. Sound pressure levels in the nearfield for such jets are generally in excess of $160 \mathrm{~dB}$ in the nearfield. Note also that the feedback shock waves (occurring outside the flow region) appear to originate at the end of the third shock-cell.

In the rest of this section we will focus on the advantages of using the LCPDI technique to study such flows that produce a highly disturbed environment.

For the interferometry measurements two convergent nozzles were used: a circular nozzle with an inner diameter of $4.5 \mathrm{~mm}$, and a rectangular nozzle with inner dimensions $1.27 \times 6.10 \mathrm{~mm}$. The rectangular nozzle was oriented with its long axis parallel to the optic axis. Compressed air was used as the gas; its total pressure was 32.0 psig (ambient pressure was $14.5 \mathrm{psig}$ ) for the circular nozzle and $60.8 \mathrm{psig}$ for the rectangular nozzle. There was a substantial pressure loss in each nozzle, so these pressures corresponded to $23.56 \mathrm{psig}$ and $53 \mathrm{psig}$ at the exit plane for the two jets as determined by a later measurement of exit pressure vs. tank pressure using a pitot probe $(0.8 \mathrm{~mm} \mathrm{o.d}$.) at the exit 
plane. The fully expanded jet Mach number was 1.26 and 1.66 for the circular and rectangular nozzles, respectively.

Figure 5 shows the results for both the LCPDI and the Mach-Zehnder interferometers. The gray scale images are coded so that darker grays indicate lower density. In both cases three shockcells are clearly present. The LCPDI has the uniform background that one would expect for this flow. The background in the Mach-Zehnder data shows a periodic structure that is characteristic of phase stepping errors indicating that the reference beam did not remain unchanged during the data acquisition. This figure clearly shows the improved performance of the LCPDI's common path design. The data reduction was automated by the phase stepping process.

The integrated density distribution represented by Eqn. 2 for the circular and rectangular nozzles are shown in Figure 6 (a) and (b). No smoothing has been done on this data, but the images were cropped down to $500 \times 350$ pixels to more clearly show the features of interest. Both images are shown with the same gray scale; the rectangular jet has a much larger density variation than the circular. The Mach diamonds are clearly visible in both images as the regions of darkest color (lowest density).

Cross sections along the centerline from both data sets are shown in Figure 7. This data was averaged over a neighborhood of 7 pixels. Again, this is the line-of-sight integral of density divided by optical path so the vertical axis is best interpreted as an arbitrary scale. Both data sets are plotted to the same scale. The flat line at the left in both cases represents the nozzle with flow from left to 
right. From this plot the shock spacing for each nozzle can be determined. The spacing for the rectangular nozzle is $4.40 \mathrm{~mm}$ and for the circular nozzle $4.32 \mathrm{~mm}$.

Seiner \& Norum ${ }^{11}$ measured the shock-cell spacing from circular nozzles that had various design mach numbers and provided a simple correlation for their data. Their correlation agreed reasonably with the theoretical wavelength derived by Pack ${ }^{12}$. For a circular jet with a design Mach number of 1 , Norum \& Seiner ${ }^{11}$ showed that the average shock spacing can be represented by $L / D=a$ $\left(M_{j}^{2}-1\right)^{b / 2}$, where $M_{j}$ is the fully expanded jet Mach number, $a=1.16$ and $b=1.0$. The shock-cell spacing $(\mathrm{L} / \mathrm{h})$ for rectangular jets can be calculated using models developed by $\mathrm{Tam}^{13}$ and Morris et al. $^{14}$. Tam ${ }^{13}$ used the Prandtl approach $\left(\right.$ Pack $\left.^{12}\right)$ and considered the shock-cell system in the jet column to be bounded by a mixing layer that was approximated by a vortex sheet. The problem was then solved by eigenfunction expansion and the solution to the eigenvalue problem yielded explicit shock-cell spacing. Morris et al. ${ }^{14}$ also used the vortex sheet model but used a boundary element capable of accommodating nozzles with arbitrary cross-sections to obtain the results. A comparison between the present measured values and those of $\operatorname{Tam}^{13}$ and Morris et al. ${ }^{14}$ is given in Table 1 . Note that such linear shock-cell models are only expected to be accurate at low levels of underexpansion before the end of the first shock-cell turns into a Mach disk.

\section{CONCLUDING REMARKS}

A new, robust, phase-stepping, common-path interferometer was used to measure the density distribution in a free jet. This interferometer has superior vibration insensitivity compared to the Mach-Zehnder interferometer because of its common path design. This is useful for vibratory environments where a reference beam has to travel long distances and can be easily disturbed. The 
present technique uses a microsphere in a liquid crystal layer to locally generate a reference beam that is not easily disturbed. The LCPDI is designed to work with a single microsphere, but in practice that has been difficult to achieve. Instead, several microspheres are present, causing extraneous interference. Also, dye is required in the liquid crystal layer to attenuate the object beam. Currently this dye changes its attenuation with applied voltage, and requires more complex data processing than standard phase stepping interferometers. Work is underway to correct these defects, but even with these drawbacks the data presented here shows that the LCPDI produces useful results.

Although previous interferometry techniques had the potential to produce quantitative density data they were not very widely used because fringe-tracking methods are often tedious, subjective, and prone to errors. The present work uses a LCPDI with the phase shifting technique to generate information required to solve three different equations for the unknown phase distribution. In addition, automatic data acquisition and data reduction eliminate the "trained eye" requirements of fringe-tracking methods.

Underexpanded supersonic jets were selected for the present study because they produce very intense acoustic tones with near-field levels in excess of $160 \mathrm{~dB}$. In addition, the underexpanded jet also displays very organized and drastic density changes that were resolved in detail by the present technique for both rectangular and circular geometries. Shock spacing were also measured for two nozzles and the results compared favorably to theory.

The LCPDI is thus an easy to use interferometer that works in harsh environments, is easy to align and is capable of measuring optical wavefronts with high accuracy. This technique has the 
potential for very wide use for density measurements in research and industrial situations. Because the data acquisition time was nominally $8 \mathrm{sec}$, only steady-state flows can currently be measured. It is believed that this acquisition time can be reduced to $0.5 \mathrm{sec} / \mathrm{frame}$ by judicious application of stepping voltages.

\section{REFERENCES}

${ }^{1}$ Powell, A., "On the Mechanism of Choked Jet Noise," Proc. Phys. Soc. London. Vol. 66, 1953, pp. $1039-1056$.

${ }^{2}$ Raman, G., "Cessation of Screech in Underexpanded Jets," J. Fluid Mech. Vol. 336, 1997, pp. 6990.

${ }^{3}$ Raman, G., "Advances in Understanding Supersonic Jet Screech," Prog. Aerospace Sci. Vol. 34, No. $1 / 2,1998$, pp. $45-106$.

${ }^{4}$ Linnik, V.P., "Simple interferometer for the investigation of optical systems," Comp. Rend. De l'Acad. Des Sci. de l'URSS. Vol. 1, 1933, pp. 208-210.

${ }^{5}$ Greivenkamp, J.E., Bruning, J.H., "Phase Shifting Interferometers," Optical Shop Testing, D. Malacara Ed., John Wiley, 1992.

${ }^{6}$ Hariharan, P., Oreb, B.F., and Eiju, T., "Digital phase-shifting interferometry: a simple errorcompensating phase calculation algorithm," Appl. Opt. Vol. 26, 1987, pp. 2504-2506. 
${ }^{7}$ Nakamura, T. and Iwamoto, J., 1996, "A quantitative analysis of axisymmetric flow with shock waves from interferogram," Fourth Asian Symposium on Visualization (Ed. Q.D. Wei), Beijing, China pp. 235-240.

${ }^{8}$ Mercer, C.R., Creath, K., "Liquid-Crystal Point-Diffraction Interferometer for Wave-Front Measurements," Applied Optics, Vol. 35, No. 10, 1996, pp. 1633-1642.

${ }^{9}$ Vest, C.M., Holographic Interferometry, John Wiley, 1979.

${ }^{10}$ Kassemi, M., Rashidnia, N., Mercer, C.R., "Numerical and experimental visualization of oscillatory temperature and velocity fields generated by a bubble," $8^{\text {th }}$ International Symposium on Flow Visualization, Sorrento, Italy, Sept. 1-4, 1998.

${ }^{11}$ Seiner, J.M. and Norum, T.D., "Aerodynamic Aspects of Shock-Containing Jet Plumes," AIAA Paper 80-00965, 1980.

${ }^{12}$ Pack, D.C., "A Note on Prandtl's Formula for the Weavelength of a Supersonic Gas Jet," Quarterly Journal of Mechanics and Applied Mathematics. Vol. 3, 1950, pp. 173-181.

${ }^{13}$ Tam, C.K.W., "The Shock-Cell Structures and Screech Tone Frequencies for Rectangular and Nonaxisymmetric Supersonic Jets," J. Sound Vi b. Vol. 121, 1988, pp. 135-147. 
${ }^{14}$ Morris, P.J., Bhat, T.R.S. and Chen, G., 1989, "A Linear Shock-Cell Model for Jets of Arbitrary Exit Geometry," J. Sound Vib. Vol. 132, pp. 199-211. 


\begin{tabular}{|c|c|c|c|c|}
\hline Nozzle & $\begin{array}{c}\text { Total Pressure } \\
(\mathrm{psig})\end{array}$ & \multicolumn{2}{|c|}{$\begin{array}{c}\text { Normalized Shock Spacing } \\
(\mathrm{L} / \mathrm{D}, \mathrm{L} / \mathrm{h})\end{array}$} & \% Difference \\
\hline & & Experimental & Calculated & \multirow{2}{*}{6.25} \\
Circular & 23.56 & $0.96 \pm 0.02$ & 0.90 (Seiner) & 0.43 \\
Rectangular & 53.0 & $3.46 \pm 0.06$ & 3.475 (Tam) & 3.90 \\
Rectangular & 53.0 & $3.46 \pm 0.06$ & 3.325 (Morris) & \\
\hline
\end{tabular}

Table I. Calculated and Measured Shock-Spacings. 


\section{CAPTIONS FOR FIGURES}

Figure (1) Typical narrowband spectrum for an underexpanded jet showing the three distinct components of supersonic jet noise.

Figure (2) Schematic of Liquid Crystal Point Diffraction Interferometer (LCPDI).

Figure (3) Experimental configuration for simultaneous LCPDI and Mach-Zehnder data acquisition.

Figure (4) Spark-schlieren photograph showing an underexpanded screeching jet at $\mathrm{M}_{\mathrm{j}}=1.4$.

Figure (5) Line-of-sight integrated density distribution downstream of a circular jet measured with (a) LCPDI (b) Mach-Zehnder interferometer.

Figure (6) Measured line-of-sight integrated density for (a) circular and (b) rectangular jets. Flow is from left to right.

Figure (7) Line-of-sight integrated density along center plane for circular and rectangular underexpanded jets. 


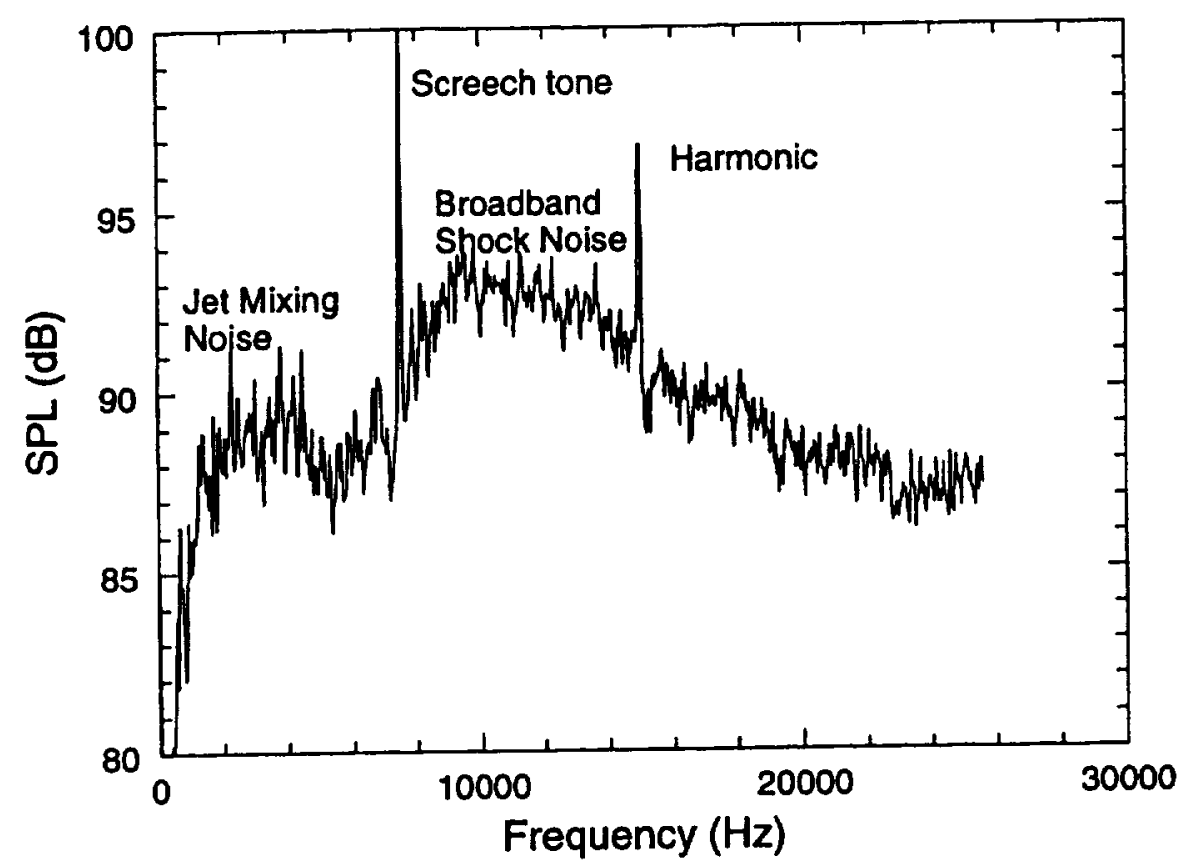

Figure 1.-Typical narrowband spectrum for an underexpanded jet showing the three distinct components of supersonic jet noise. 


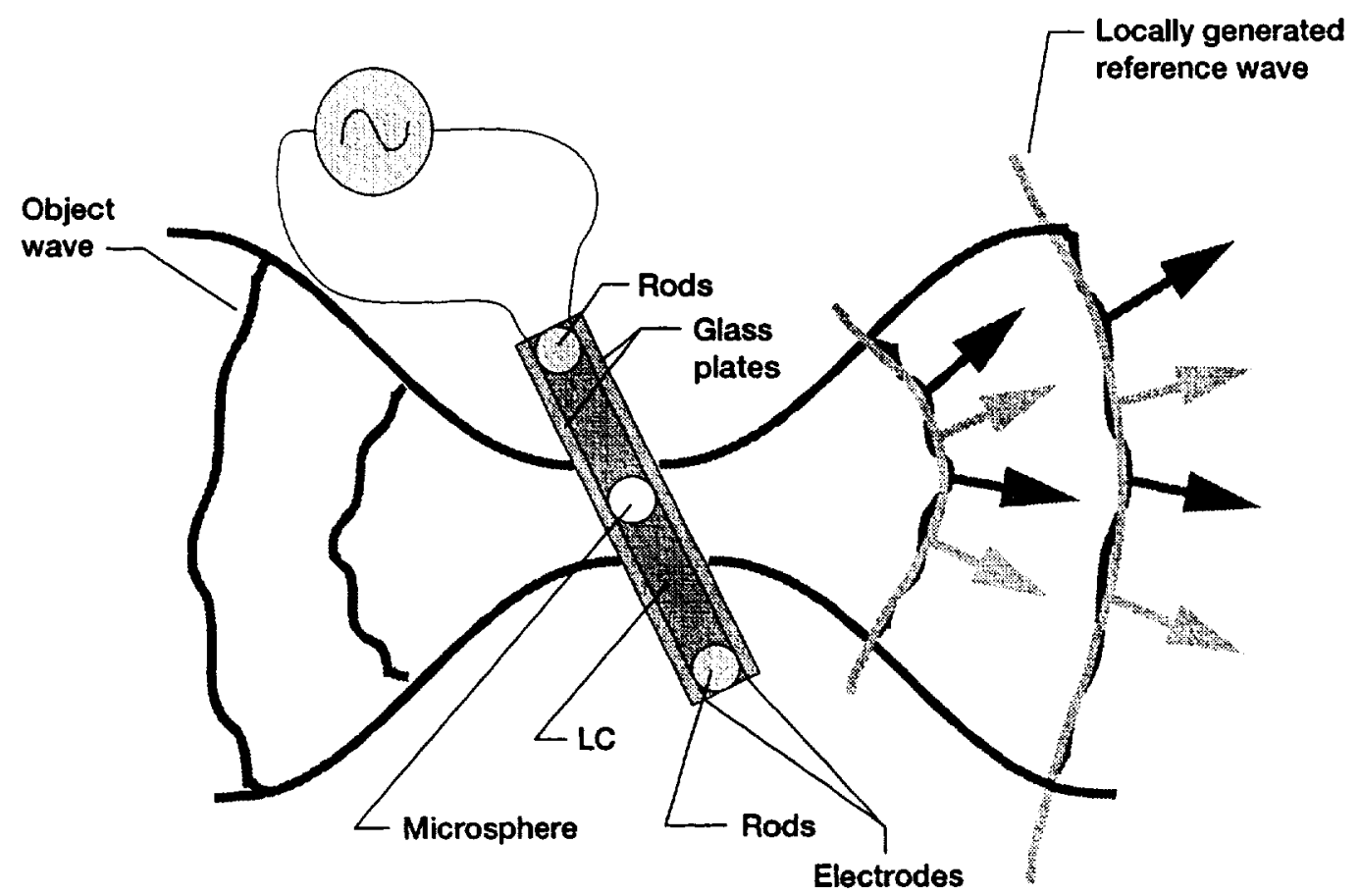

Figure 2.-Schematic of Liquid Crystal Point Diffraction interferometer (LCPDI). 


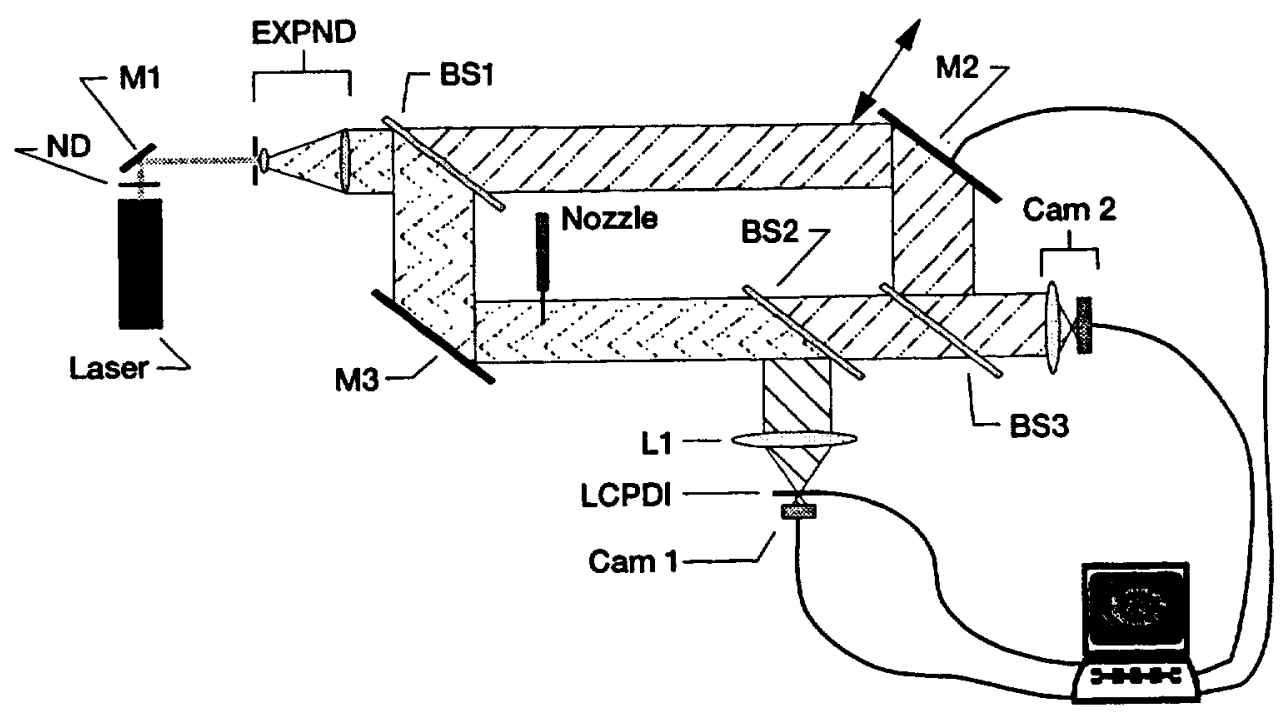

Figure 3.-Experimental configuration for simultaneous LCPDI and Mach-Zehnder data acquisition. 


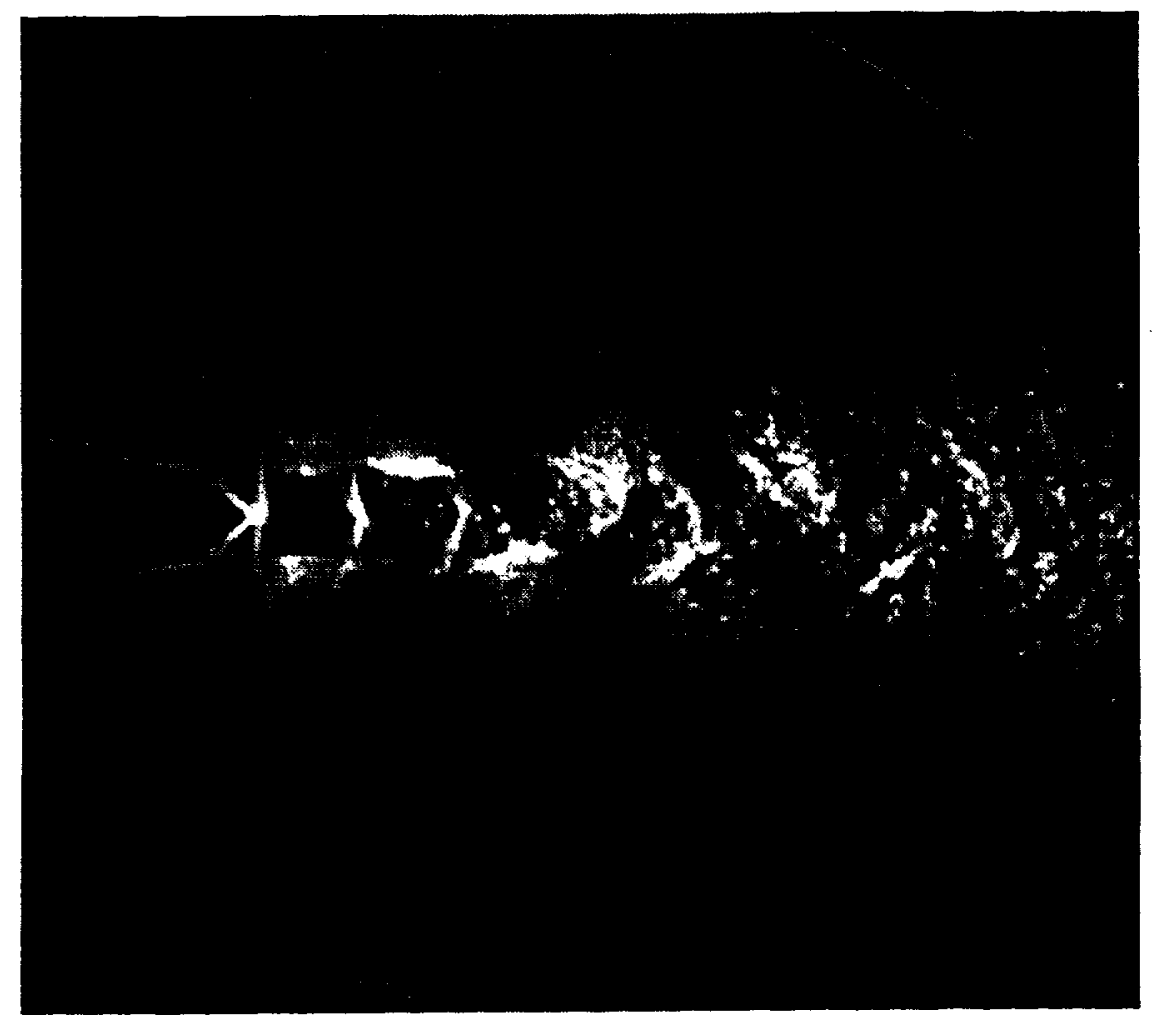

Figure 4.-Spark schlieren photograph showing an underexpanded screeching jet at $M_{j}=1.4$. 


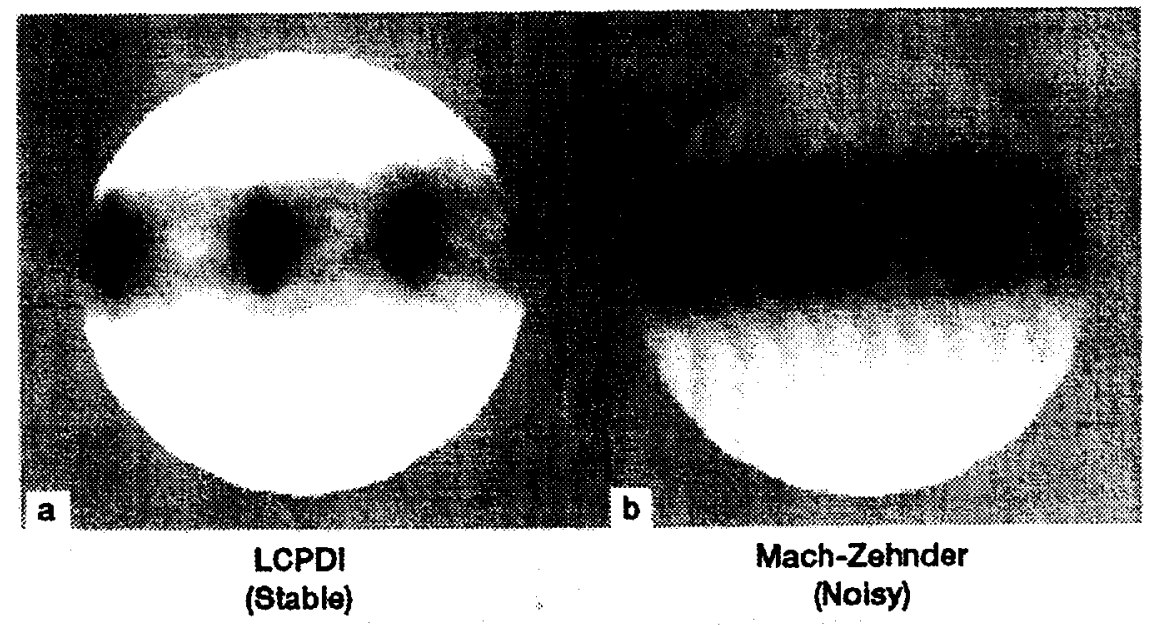

Figure 5.-Line-of-sight integrated density distribution downstream of a circular jet measured with (a) LCPDI. (b) Mach-Zehnder interferometer. 

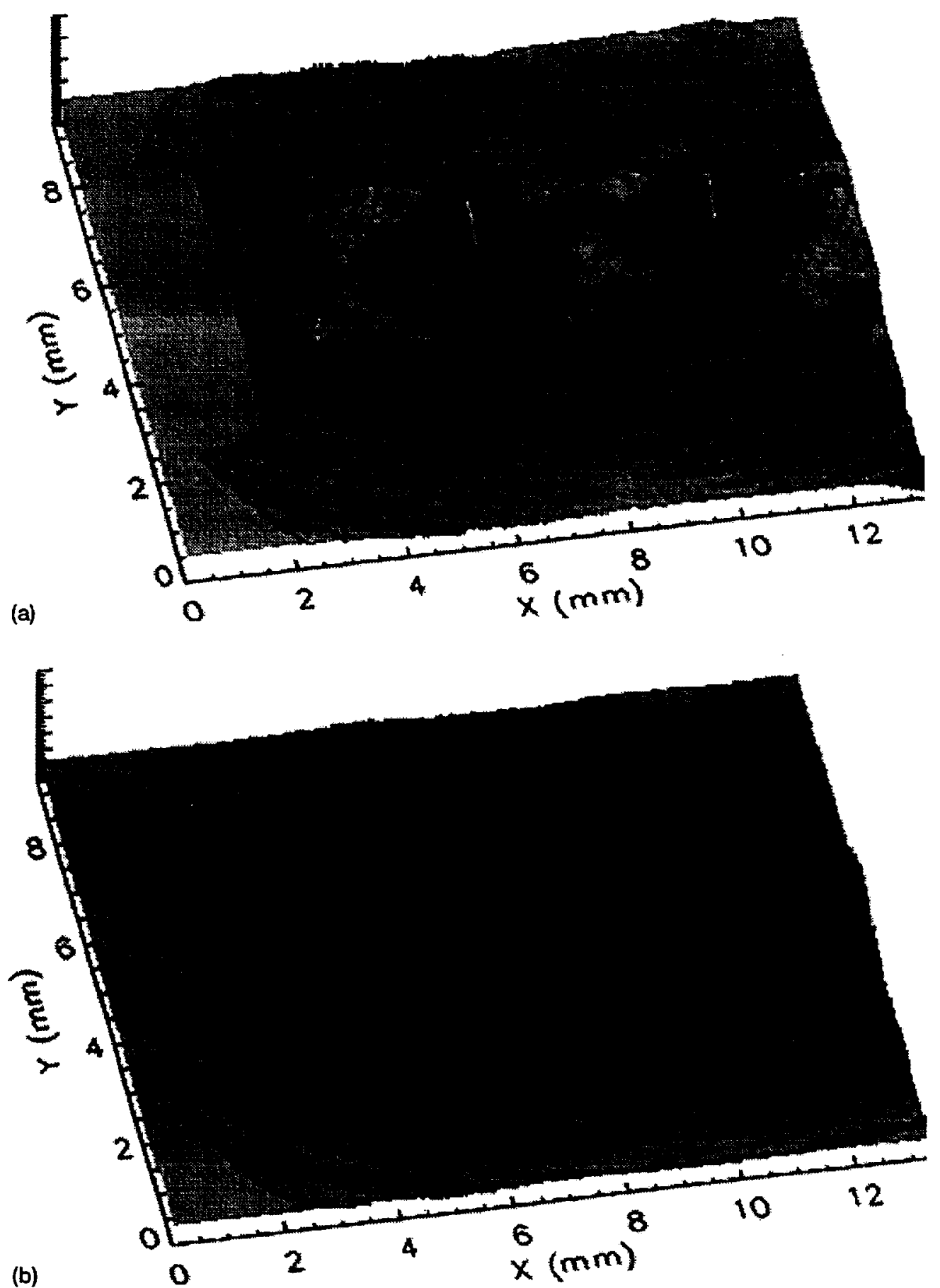

Figure 6.-Measured line-of-sight integrated density for (a) circular and (b) rectangular jets. Flow is from left to right. 


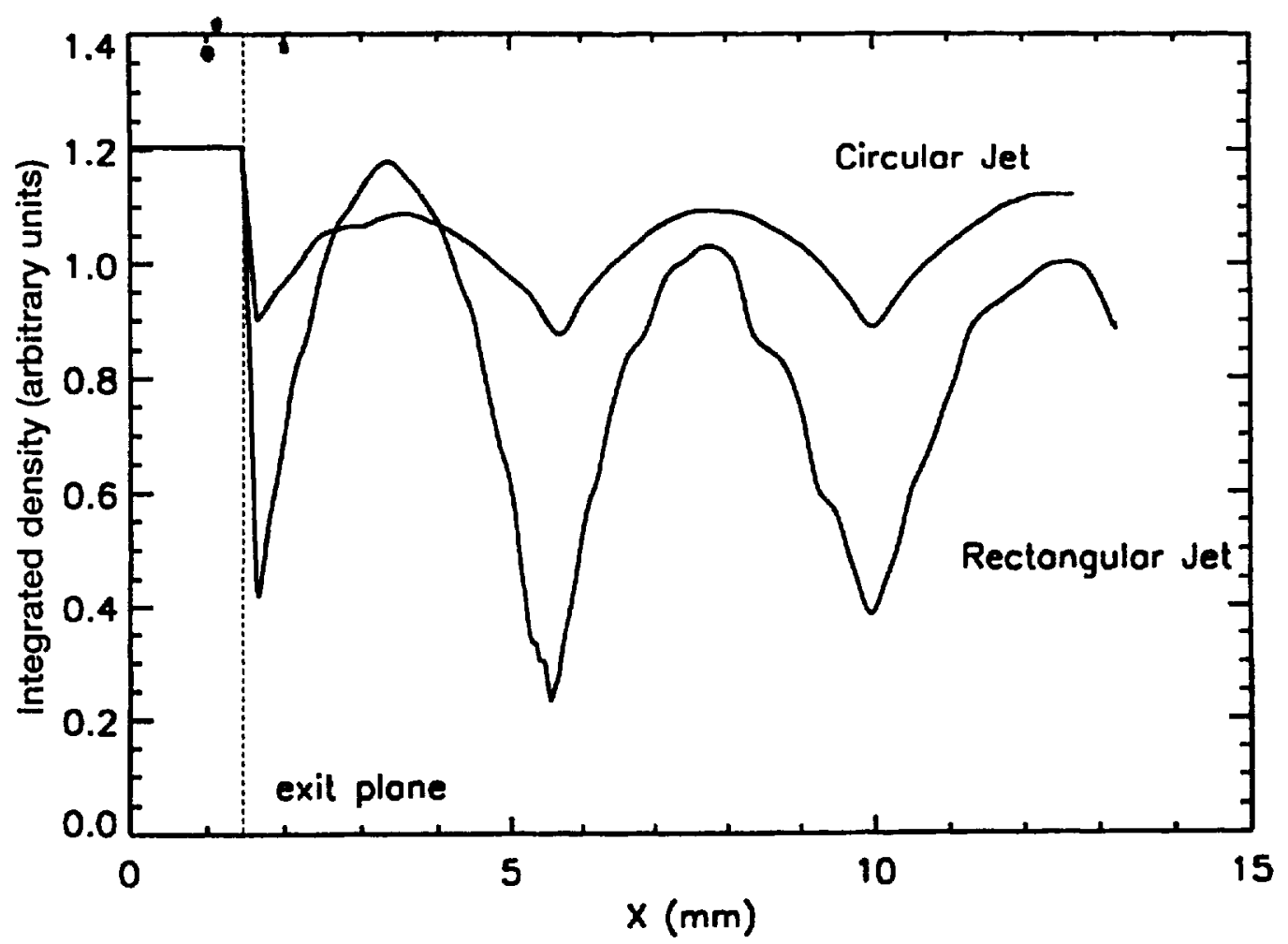

Figure 7.-Line-of-sight integrated density along center plane for circular and rectangular underexpanded jets. 
